\title{
Promotion of Physical Activity Through Reablement for Older Adults: Exploring Healthcare Professionals' Clinical Reasoning
}

\author{
Hanne Leirbekk Mjøsund (D) \\ Cathrine Fredriksen Moe (D) ${ }^{1,2}$ \\ Elissa Burton (iD ${ }^{3}$ \\ Lisbeth Uhrenfeldt (D) \\ 'Faculty of Nursing and Health Sciences, \\ Nord University, Bodø, Norway; \\ ${ }^{2}$ Nordland Hospital Trust, Bodø, \\ Norway; ${ }^{3}$ Curtin School of Allied Health, \\ Curtin University, Perth, Australia
}

Correspondence: Hanne Leirbekk

Email hanne.I.mjosund@nord.no
Introduction: Reablement is an interdisciplinary, multifactorial, and individualized intervention aimed at improving function and maintaining the independence of communitydwelling older adults who receive home care services. Physical activity (PA) is important for functional ability in older adults, but it is unclear how PA is promoted through reablement. Healthcare professionals' (HCPs) clinical reasoning and decision-making are essential and determine how reablement is delivered to individuals. Exploring how HCPs integrate PA into their clinical reasoning is critical to understanding how PA is integrated within reablement. To gain knowledge of how PA is integrated within reablement, there is a need to explore how HCPs integrate PA into their clinical reasoning.

Purpose: The study aimed to explore how PA is integrated into HCPs' clinical reasoning in a Norwegian reablement setting.

Methods: Sixteen HCPs, including occupational and physical therapists, registered nurses, and other home care staff, were recruited from four Norwegian municipalities. They participated in semi-structured interviews that were transcribed verbatim, and an interpretive content analysis approach was used.

Results: PA was integrated into multifaceted clinical reasoning captured by the main theme: "Improving the person's ability to participate in meaningful activities." Within this overall theme, two sub-themes emerged with a primary focus on either i) increasing physical capacity or ii) improving activity performance. Each subtheme encompassed different aspects of clinical reasoning and diverse perspectives on how to integrate PA in reablement. Conclusion: HCPs' decision-making in reablement builds upon complex clinical reasoning and incorporates diverse perspectives on integrating PA in the delivery of reablement. This broad approach may be useful in targeting different needs, preferences, and contexts. There is a need to further investigate how PA is appropriately promoted through reablement, how it meets the needs and preferences of participants, and which contextual factors influence PA promotion through reablement.

Keywords: health services of the aged, interdisciplinary research, exercise therapy, health knowledge, patient-centered care, activities of daily living

\section{Introduction}

Emerging in several industrialized countries over the last two decades, reablement is an interdisciplinary home care approach that aims to improve function and maintain independence among people receiving home care services. ${ }^{1}$ There has been high political interest in reablement due to its promising expectations for 
reducing healthcare spending and solving some of the sustainability challenges found within an aging population. $^{2}$

Internationally, reablement is provided either through social care services or healthcare services and involves various interventions that builds upon goal-oriented support plans. ${ }^{1}$ Reablement is not specific to particular diseases, and those receiving reablement are typically a heterogenic group comprised of older adults with a mean age of 80 years $^{3}$ and no severe cognitive problems ${ }^{4-6}$ who are experiencing challenges with managing their daily life independently. ${ }^{1}$ In Norway, where this study was conducted, reablement is provided as part of the publicly-funded municipal healthcare service. It may be organized differently between municipalities, but is typically handled by the pre-existing home care service or a specialized reablement team. ${ }^{7}$ Within the reablement team, occupational therapists (OTs) and physical therapists (PTs) often are described as consultants and/or advisors who are responsible for developing, supervising, and adjusting a reablement plan, while home care staff (with or without formal education) are responsible for delivering reablement. ${ }^{8,9}$ In the following study, the term healthcare professionals (HCPs) is used as a common term for all personnel delivering reablement, regardless of educational background.

Despite its emerging popularity, the scientific evidence of the effect of reablement for improving function and independence remains limited, ${ }^{10-12}$ and there is insufficient knowledge about which components are critical for successful outcomes. ${ }^{10}$ The international classification of functioning (ICF) outlines three levels of functioning, including body functions and structures (eg strength or balance), activity (ie the execution of a task) and participation (ie involvement in a life situation). ${ }^{13}$ In a recent definition of reablement developed through a Delphi study with reablement experts, it suggests that reablement consists of training in daily activities, home modifications, assistive devices and involvement of a social network. ${ }^{1}$ While these intervention components may target the levels of activity and participation, none of them specifically targets body functions, which may influence the effectiveness of reablement.

Physical activity (PA) is important for improving and maintaining body functions and structures in older adults and influences their ability to manage activities of daily living (ADL), ${ }^{14}$ reducing the risk of falling, ${ }^{15}$ and decreasing frailty. ${ }^{16} \mathrm{~A}$ recent scoping review showed that the integration of PA in reablement varies and that it is unclear how the promotion of PA is prioritized among other reablement interventions. ${ }^{3}$ While the majority of studies published over the last two decades included exercises as a component of reablement, as well as a focus on practicing and participating in ADLs, ${ }^{3}$ there is no consensus that exercises or motivation for PA should be included in reablement. $^{1}$

Global and nation-specific health policy strategies recommend that older adults be physically active with moderate intensity for at least 150 minutes a week in addition to completing activities that target strength and balance and reduce sedentary behavior. ${ }^{17,18}$ PA may consist of a range of different activities, such as structured and timely planned exercise, leisure time activities, transportation, household tasks, and other everyday activities. Importantly, PA should be adjusted to functional levels, with the understanding that doing some PA is better than doing none. ${ }^{18}$ Strategies from the World Health Organization (WHO) emphasize the importance of implementing evidence-based actions and ensuring that HCPs provide simple and timely advice about PA and sedentary behavior tailored to individual health needs, capacity, and preferences. $^{19}$

However, the task of promoting PA among older adults is complex and challenging, and PA levels generally decrease with age, particularly among people who require assistance from others. ${ }^{20}$ Older adults receiving home care report several barriers that prevent them from being physically active, such as injury or illness, a feeling of being too old, or a lack of social support. ${ }^{21}$ Older adults receiving PA interventions report that HCPs play an important role in their experiences related to PA. They find that the HCPs' delivery of PA interventions is as important as the content of these interventions, ${ }^{18}$ emphasizing the importance of being able to see the value of PA as well as experiencing it as enjoyable. ${ }^{22}$

In the context of reablement, decision-making should be person-centered (here, the term "participant" will be used for people receiving reablement) and targeted toward individual goals set by the participants themselves. ${ }^{1,23-25}$ To develop an evidence-based approach, the HCPs need to integrate different types of knowledge including research evidence, information from their own experiences and expertise, and awareness of the individual participant, including contextual factors to ensure feasibility, appropriateness, meaningfulness and effectiveness. ${ }^{26}$ Thus, promoting PA not only depends on HCPs knowing the 
benefits of PA but also on how they utilize, collate, and prioritize what they know in their clinical reasoning and decisions. ${ }^{27}$ For this study, we consider clinical reasoning to be

the thinking and decision-making processes associated with clinical practice [...] that allows practitioners to make difficult decisions in the conditions of complexity and uncertainty that often occur in health care. ${ }^{27}$ p. 3

Although clinical reasoning is often associated with the thinking processes based on knowledge within a particular profession (typically those requiring a bachelor's degree), in this study, we hold that clinical reasoning involves the thinking and decision-making of all HCPs involved in reablement.

The interdisciplinary nature of reablement makes it possible to combine knowledge from several disciplinary fields, such as theories of occupational performance, ${ }^{4}$ motor learning theory, ${ }^{28}$ and caring science. ${ }^{29}$ This interdisciplinarity presents opportunities to bring together complementary perspectives and knowledge, relying on interdisciplinary collaboration with respectful negotiation and shared decision-making between HCPs. ${ }^{23,30,31}$ To gain a better understanding of how HCPs promote PA in reablement, research needs to explore how PA is integrated into HCPs' reasoning processes in a reablement setting. This study aimed, therefore, to explore how PA is integrated into HCPs' clinical reasoning in a Norwegian reablement context.

\section{Design and Methods}

Inspired by a realist perspective, the study aims to foster an understanding of patterns and mechanisms that may explain how and why reality unfolds as it does in a particular context. ${ }^{32}$ The study uses a qualitative exploratory design based on individual interviews. The consolidated criteria for reporting qualitative research (COREQ) was used to ensure that the relevant study information was reported. $^{33}$

\section{Sampling Strategy and Recruitment}

A purposive sampling strategy was used based on the principles of variation sampling, which is targeted toward gaining variation in small samples based on pre-defined selection criteria. ${ }^{34}$ We included HCPs with diverse professional backgrounds who were central in reablement delivery in their municipality. The HCPs were recruited from municipalities that differed in size and organizational model because these factors could involve different premises for practice. ${ }^{7,35}$ By including this heterogeneity in the study sample, we aimed to learn the central themes that cut across the existing variation and capture diverging perspectives related to the phenomena being explored. ${ }^{34}$

We selected eligible municipalities and asked the leaders of the reablement teams in these municipalities for permission to contact potential candidates from their teams. We encouraged the leaders to suggest potential candidates whom they considered to be reflective about their practice, who had at least one year of experience with reablement, and who represented diverse professional groups. We contacted each potential candidate either by phone or e-mail, provided oral and written information about the study, and then asked if they were willing to participate. All the reablement leaders we contacted reacted positively, and all the HCPs who were recommended and contacted agreed to participate.

\section{Study Sample and Setting}

Sixteen HCPs from four municipalities were included in this study, including four OTs, four PTs, four home care assistants, two registered nurses (RNs), and two HCPs with other educational backgrounds. The HCPs' median age was 46.5 (ranging from 29 to 57), and two of them were male. On average, they had 19 years of professional experience (ranging from four to 33) and four years of experience working with reablement (ranging from one to six years). Most HCPs $(n=15)$ had additional education/ courses beyond their basic education, eg, reablement, rehabilitation and habilitation, geriatrics, supervision, and motivational interviews, and two of them had master's degrees. Further characteristics of the participants are presented in Table 1. The HCPs were recruited from four Norwegian municipalities ranging in size from $\sim 4000$ to $\sim 200,000$ people. Two of the municipalities had organized reablement into specialized teams, while two municipalities provided reablement as an integrated part of the existing home care services. The duration of the reablement interventions in all municipalities was approximately six weeks with exceptions if needed. Visit frequency and duration varied between municipalities, ranging between 2-5 visits per week and 20-60 minutes per visit. Additional characteristics of the reablement organization in each municipality are presented in Table 2 . 
Table I Main Characteristics of HCPs

\begin{tabular}{|l|l|l|l|l|l|}
\hline $\begin{array}{l}\text { Healthcare } \\
\text { Personnel }\end{array}$ & N & $\begin{array}{l}\text { Gender (Male/ } \\
\text { Female) }\end{array}$ & $\begin{array}{l}\text { Age, Mean } \\
\text { (Range) }\end{array}$ & $\begin{array}{l}\text { Years of Professional Experience, } \\
\text { Mean (Range) }\end{array}$ & $\begin{array}{l}\text { Years of Experience with } \\
\text { Reablement, Mean (Range) }\end{array}$ \\
\hline OTs & 4 & $1 / 3$ & $36(29-43)$ & $11.5(6-17)$ & $4.5(4-5)$ \\
PTs & 4 & $1 / 3$ & $5 I(40-56)$ & $24(17-31)$ & $4(1-6)$ \\
RNs & 2 & $0 / 2$ & $51(44-57)$ & $17.5(4-31)$ & $3.5(3-4)$ \\
Home care & 4 & $0 / 4$ & $54(49-56)$ & $30.7(29-33)$ & $4.5(4-6)$ \\
assistants & 2 & $0 / 2$ & $33(30-35)$ & $9.5(7-12)$ & $3.5(3-4)$ \\
Other & 2 & & & \\
\hline
\end{tabular}

Abbreviations: OT, occupational therapist; PT, physical therapist; RN, registered nurse.

\section{Data Collection}

We developed a semi-structured interview guide and piloted it with HCPs working with reablement in a municipality not included in the study (see Supplementary file 1). The questions in the guide were designed to encourage the HCPs to provide rich descriptions of their experiences and clinical reasoning related to $\mathrm{PA}$ and to provide information about how they integrated PA into their general clinical reasoning in reablement. The guide included guidance for conversational topics and interview directions, but the order of questions was not followed strictly.

Face-to-face individual interviews were conducted and audio-recorded between May and October 2019 in a quiet office or meeting room at the HCP's workplace. Each interview lasted 70-90 minutes and was undertaken by the first author, who had completed interviewing classes, practice and supervision prior to interviewing. Field notes were made after each interview to note first impressions and experiences in the interviews. The interviewer had no relationship to the HCPs prior to study commencement, but introduced her own professional background and aim of the research prior to the interviews. After analyzing 16 interviews, we found the data to be sufficiently saturated to tell a rich, complex, and coherent story addressing our research question, so there was no need to recruit additional informants or undertake follow-up interviews. ${ }^{36}$

\section{Data Analysis}

We used an inductive qualitative content analysis approach to interpret the meaning of the data. ${ }^{37}$ All interviews were transcribed verbatim and read several times to gain a better understanding of the meaning of the text. We searched each transcript for meaningful units of text that showed the HCPs' clinical reasoning about PA, which were then extracted and condensed, using NVivo software $\mathbb{C}$. The condensed text units were organized into codes, categories, two sub-themes, and one main theme, following the hierarchy presented by Erlingsson and Brysiewicz ${ }^{37}$ and illustrated by the conceptual map in Figure 1. While the organization of the codes and categories was mainly based on the manifest content of the data, the development of sub-themes and the main theme was an interpretational process, elaborated on by reflective thinking and by critical questioning of how the themes developed in accordance with the data. The research team critically discussed the interpretations, questioning and reflecting

Table 2 General Characteristics of Reablement Organization in Each Municipality

\begin{tabular}{|l|l|l|l|l|}
\hline & Municipality I & $\begin{array}{l}\text { Municipality } \\
\mathbf{2}\end{array}$ & Municipality 3 & Municipality 4 \\
\hline $\begin{array}{l}\text { Duration of reablement } \\
\text { interventions }\end{array}$ & $\begin{array}{l}3-10 \text { weeks, average 6 } \\
\text { weeks }\end{array}$ & Mainly 6 weeks & $6-8$ weeks & $\begin{array}{l}\text { Most often }<6 \\
\text { weeks }\end{array}$ \\
\hline Visits per week & 2 & $2-5$ & $2-3$ & 5 \\
\hline Duration per visit & $30-60$ min & 20 min & $\begin{array}{l}60 \text { min (often more in the } \\
\text { beginning) }\end{array}$ & 60 min \\
\hline Organizational model & Integrated & Integrated & Specialized team & Specialized team \\
\hline
\end{tabular}

Note: Information based on the HCPs' descriptions. 


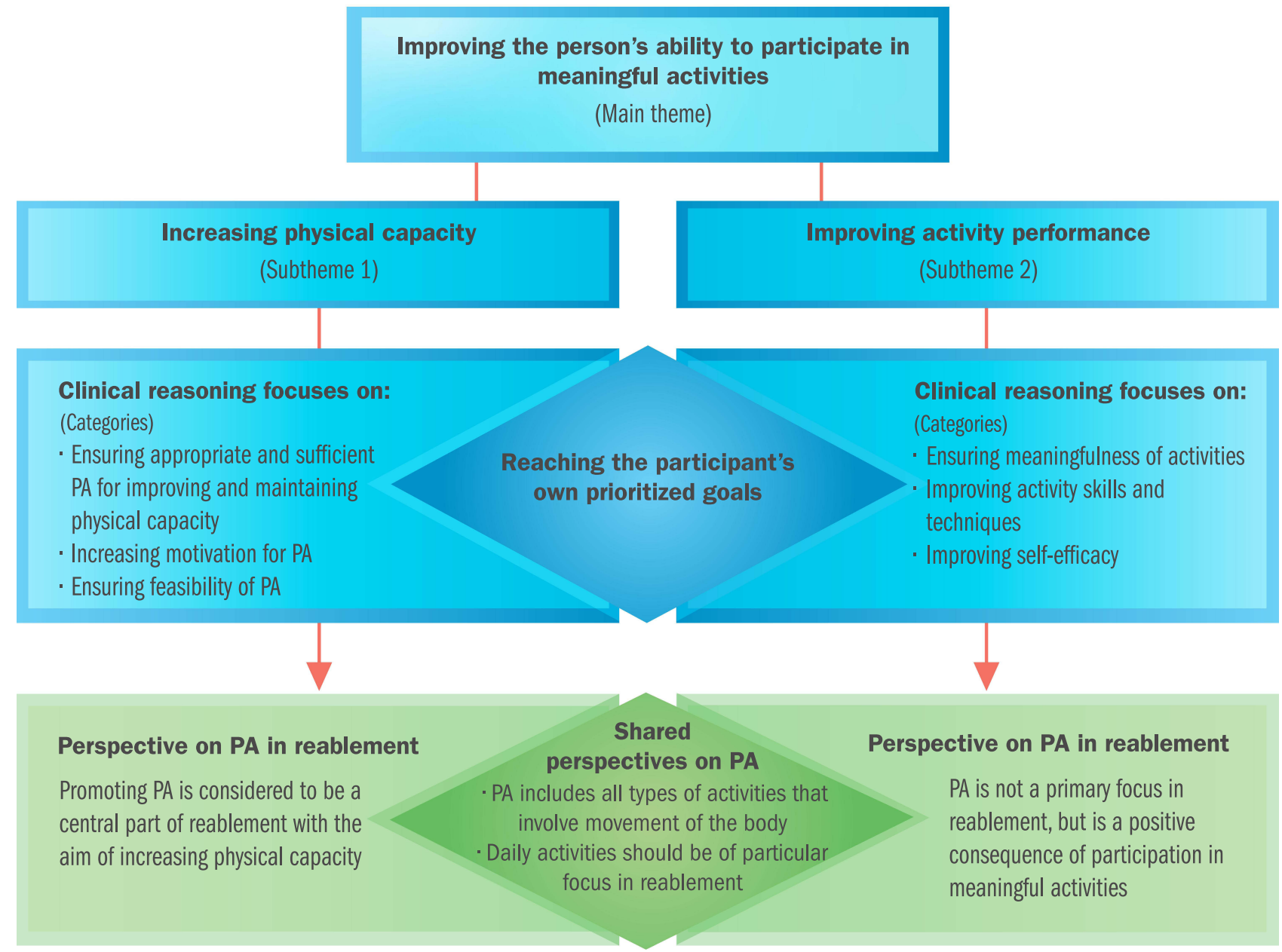

Figure I Conceptual map illustrating the healthcare professionals' clinical reasoning and perspectives on PA.

Abbreviation: PA, physical activity.

on the coherence of the analysis and how the researchers' pre-understandings influenced it. The analysis was not linear but followed a continuous and flexible reflective process that compared content in parts of the data with interpretations of the whole data. ${ }^{37}$

We enhanced the trustworthiness of the study methods by carefully considering questions raised in the checklist by Elo et al. ${ }^{38}$ To enhance the transparency of the findings, we selected quotes from the participants that exemplified the connections between the empirical data and the results. $^{38}$

\section{Results}

The HCPs' clinical reasoning was multifaceted and reflected in the overarching theme "improving the person's ability to participate in meaningful activities," in which the participants' own prioritized goals were central to the HCP's reablement strategy. There was agreement among HCPs that PA involved all types of bodily movement and participation in daily activities was a particular focus in reablement. Within this overarching theme, two subthemes arose, with a primary focus on either 1) increasing physical capacity or 2) improving activity performance. Each of the subthemes involved different aspects of clinical reasoning as well as diverse perspectives on how PA should be integrated into reablement. Within the first subtheme, the HCPs believed that promoting PA to increase physical capacity was a central part of reablement and their clinical reasoning had a primary focus on i) ensuring appropriate and sufficient PA to improve and maintain physical capacity, ii) increasing motivation for PA, and iii) ensuring the feasibility of PA. Within the second subtheme, the HCPs did not consider PA to be a primary focus in reablement, but rather, they saw it as a positive consequence of participating in meaningful activities. Within this subtheme, the HCPs' 
clinical reasoning was primarily focused on i) ensuring the meaningfulness of activities, ii) improving activity skills and techniques, and iii) improving self-efficacy. A conceptual map illustrating the themes, subthemes, and categories, as well as the different perspectives on how PA should be integrated into reablement, is provided in Figure 1.

\section{Subtheme I: Increasing Physical Capacity}

Within subtheme one, the HCPs considered reduced physical capacity, such as diminished strength, balance, and endurance, to be a central cause of the participants' functional challenges. They perceived that the participants' lack of physical capacity made them feel unsafe, which become a barrier to their taking part in daily life activities. Another perception was that participants lacked energy, which could lead to increased sedentary behavior and further reduced their physical capacity and function. Thus, HCPs believed that increasing the participants' PA levels was critical for improving their physical capacity and reaching their reablement goals.

When we exercise [...], it is exercises in order to be able to do another activity, right? It is like the brick wall, which enables you to come out from your house, down to the bus, and down to your target. [...] So the exercises themselves are not the goal. The exercises are part of the way towards the goal. (PT 2)

\section{Ensuring Appropriate and Sufficient PA for Improving and Maintaining Physical Capacity}

The HCPs considered the types, intensities, and amount of PA needed to improve and maintain physical capacity, though in careful balance with the participants' motivation, goals, and contextual premises. They believed participation in daily activities to be an important type of PA and encouraged the participants to complete tasks such as cleaning the house, going to social events, or walking stairs.

We really emphasize the everyday activities. [...] Perhaps we see that continuing to vacuum, doing the laundry and things like that is so important for your physical health. (PT 1)

In most cases, the HCPs found it necessary to also introduce specific exercises to help the participants improve physical capacity. The HCPs emphasized that the exercises they used in reablement were evidence-based and would typically include a set of four simple exercises that they knew, through research, to be beneficial for older adults, and sometimes adding a few other simple exercises tailored to the participant's individual needs.

First of all, it needs to be some exercises that I know have a documented effect on what we aim for. If you want to become stronger in your legs, then it needs to be some exercises that have been researched and show that you do actually become stronger in your legs by doing this. (PT 2)

The HCPs focused on facilitating the sufficient amount, intensity, and quality of PA. This could involve pushing the participants to give an extra effort in the exercise activities or encouraging them to do additional exercises on their own time. It also meant ensuring that the participants performed the exercises or PA with a good technique to improve the perceived effect. For example, this might include teaching the participants to rise from a chair correctly (working toward not using their hands) or walking stairs with an upright posture.

When you are going to sit down, many people just let themselves fall down. Then we need to be attentive that you ... need to sit down slowly because it strengthens the musculature in your thighs better. (RN 1)

\section{Increasing Motivation for PA}

Motivating the participants to be physically active was identified by HCPs as a crucial part of reablement. They emphasized helping participants to understand and experience the benefits of PA in managing their daily life. When promoting PA, the HCPs found it important to initially build trust with the participants and to ensure that they felt seen and heard. The HCPs also considered it crucial that the participants felt that PA was meaningful and related to their reablement goals and they described how they would try to influence the participants' views on PA, ensuring that they understood the connection between their PA behavior and their physical capacity and function.

My experience is that when you have some exercises that you are going to do with them, then it is important that ... you need to understand why you are doing this. [...] You need to give an explanation ... what happens with your body when you do this and this, and why is this important. (Home care assistant 2)

The HCPs perceived that insecurity and the fear of falling often were barriers for being physically active among the participants. Thus, they considered how they could make participants feel safe during PA and assure them that it was not hazardous for their health condition. Informing and reassuring participants that it was normal to experience 
muscular soreness, pain, increased heart rate, or breathlessness was important.

And it is a lot about explaining this to the participants, so that ... it is not dangerous to walk, to have a high pulse [...]. One may think it is a bit scary to increase the pulse [...]. Many also become sore, that have not been sore in many, many years, right. [...] Then it is about explaining to them in a professional, reasonable way. (OT 4)

A priority for HCPs was balancing the intensity and degree of exercise challenge, so that participants experienced a feeling of success and self-efficacy while still being challenged sufficiently to make progress. They described how they motivated the participants to give an extra effort by doing the exercises with them, making the exercises fun and enjoyable, challenging them to improve their physical test results, counting repetitions along the way, and pushing them to give a little bit more. The HCPs perceived that a rapid improvement at the beginning of reablement often led to improved energy and motivation to re-engage in activities that they had previously discontinued: "To get them over the hill where they feel it, that it does good, that it is helpful for them" (PT 1). The HCPs believed it is important to point out and explain any improvements and found that reassessments of physical tests were useful in visualizing improvements and reinforcing both the participants' and the HCPs' beliefs that their efforts were worthwhile.

When they are to take a new test, then you can see how much faster they can walk or how much stronger they have become. That motivation is really good. (RN 1)

\section{Ensuring the Feasibility of PA}

The HCPs emphasized that the PA and exercises they recommended were feasible within the context of reablement and the participants' daily life. The PTs typically were responsible for planning the exercise interventions. They described how they recommended exercises that were familiar to the home trainers and easy to perform and supervise, rather than suggesting more individually targeted exercises, which would have been preferred if they were able to follow up on the exercises themselves. When following up on the exercises, the home trainers would focus on observing the participants' day-to-day function and health status, considering if any adaptations were needed.
It needs to be feasible for the home care staff [...] it needs to be a bit easy and easily understood, so that everyone from the home care service that visits are able to instruct on these exercises [...] because if you make a program [...] that, per definition, should be the most optimal program in the world. But if it just ends up in a drawer or among the newspapers because it is hopeless to carry it out, both for the participant and for those who shall instruct it, then it's a waste. The best then becomes the enemy of the good. (PT 2)

Adapting PA to the participants' daily environments, interests, and habits was key for the HCPs, and they focused on how to foster the participants' desire to maintain their PA habits after reablement. Activities such as stair walking or outdoor walking were considered particularly suitable because these activities were easier for the participants to undertake and related to their everyday living, yet were considered effective for increasing physical capacity.

How can we help them establish good habits so they can maintain their function when we are done? To make ourselves redundant, that is the most important part. (PT 4)

\section{Subtheme 2: Improving Activity Performance}

Within the second subtheme, the HCPs emphasized that the reablement interventions should consist of practicing the participants' goal activities, believing that the limitations in skills and self-efficacy were the core reasons for activity challenges among the participants. Within this subtheme, the activity performance was the central consideration, rather than the amount of PA involved in activities. The HCPs considered PA to be a positive consequence of participating in daily activities, rather than a primary focus in reablement.

They [the participants] may expect that they are going to exercise, in the old-fashioned way [...]. You need to work a bit with the part that ... it is actually the activity you want to do, that's where we need to practice. So if you are going to the town with your wheeled walker, that's what we need to practice. (OT 2)

\section{Ensuring Meaningfulness of Activities}

According to the HCPs, it was essential that the participants' reablement activities be important and meaningful in their daily life. The HCPs considered the initial process of mapping the participant's daily life, interests, and 
challenges to be key in developing meaningful goals and identifying the activities they wanted to practice through reablement. Goal-setting and motivational interviews were considered helpful for the participants in identifying meaningful goals that they were motivated to work on.

We use quite a lot of time on the mapping process, we may likely have $2-3$ visits. We see that it is very valuable because it is about getting to know them and give them time. This is also a process for them in order to identify a goal. (OT 3)

The HCPs wanted to ensure that the participants can prioritize the activities they found most meaningful in their everyday life, and they would supervise the participants in how to preserve energy to participate in the activities they valued most. This could involve suggesting that activities be spread over the week or showing how they could engage in activities in new ways that did not demand as much energy.

A lot of it is about supervising about how to use your energy well. [...] If you are going to a social activity in the afternoon, then that may not be the day to take a shower because then you become so exhausted that it affects the quality of the social activity you are to do later, which is important for you. (OT 2)

\section{Improving Activity Skills and Techniques}

The HCPs described how they would carefully observe the participants performing an activity to analyze and identify challenging subtasks of the activity. They would supervise and suggest alternative techniques that could improve the participants' ability to accomplish the activity and also their feeling of safety when doing so. This could also involve introducing aids or equipment or re-arranging the furniture to alter the demands of the activity.

If someone is afraid of falling in the shower and wants to shower safely, then you need to consider what is needed for the person to shower safely. [...] You observe and let the person do it. Then you start analyzing. What did the person do and what could the person have done differently to make it more safe? Then you practice the small elements that you see. The clothes need to be within range. A shower mat may be needed. Handles. A safety alarm close by perhaps. Take your time. Perhaps sit on a stool in the shower. May need some helping aids. [...] So it is very much about technique in order to accomplish stuff. (PT 3)
It was common for HCPs to split the goal activity into subtasks or subgoals and work toward improving one part at a time. In the reablement sessions, they would emphasize practicing each subtask repeatedly until the participant felt confident performing it by themselves. When the participants were able to do the subtasks themselves, the HCPs would help the participant begin working on the next subtask, while continuing with the previous subtasks they mastered.

When a goal is accomplished, that you, as an example, manage to walk down the stairs alone, then we may agree to meet the participant down by the stairs. Then they have accomplished one of their subgoals. (Home care assistant, 2)

\section{Improving Self-Efficacy}

According to the HCPs, the participants' lack of selfefficacy is an essential barrier to taking part in daily activities. During the reablement intervention, the HCPs focused on assisting the participants in gaining feelings of success when performing activities, considering this essential to their motivation for engaging in meaningful daily activities. They emphasized that noting and commenting on any small improvements helped the participants see they were progressing.

We had one participant that was not so motivated for exercises, [...] but he had been painting. [...] And he had not done that since he had a stroke five years ago. [...] That is kind of like a physical activity as well. It did something with his self-efficacy. I think that is the strongest ... moment I have had these years. I sat behind him, and then I saw that he was completely in his own world. [...] It is about finding something that is positive. The small things they can do, that they may not have been able to before. (Home care assistant 1)

Repeated practice and gradual exposure to the activity were considered important for improving the participants' self-efficacy and confidence in undertaking activities. The HCPs perceived that insecurity, anxiety, and other psychological issues were common barriers to engaging in activities and considered it important to support the participants by being present, enabling them to try the activity, one part at a time. They emphasized that it was key that the participants felt confident in performing the entire activity in its correct environment for them to be motivated to continue doing the activities alone. 
If you are bringing your wheeled walker to the bus, then it is good to have someone along with you the first time. Because there is so much insecurity about it, there is a lot [of] [...] what should I do and how. Then we have accompanied them on the bus with their wheeled walker [...]. To see that, yes, you walk in here, then sit down there, you pay here, then sit down there, and then out again. And if you have done that once together with someone, then you have a lower threshold for doing it yourself the next time. And the chance that the goal is accomplished is higher. (OT 2)

\section{Discussion}

This study aimed to explore how HCPs integrate PA into their clinical reasoning in reablement. The findings show that although the HCPs shared common overall perspectives on reablement and PA, their clinical reasoning was diverse, with a primary focus on either increasing physical capacity or improving activity performance. The findings of this study exemplify the complex and multifactorial interdisciplinary clinical reasoning processes related to promoting PA in a real-life healthcare setting and, additionally, provides evidence that different perspectives may influence how HCPs prioritize PA promotion in their reablement delivery.

The diverging perspectives on PA found in this study share similarities with the conflicting perspectives about PA described in the Delphi study by Metzelthin and colleagues. ${ }^{1}$ Likewise, Eliassen and Lahelle found diverse practices in Norwegian municipalities, with an emphasis on either exercise-based training, activity-based training, or a combination of both. ${ }^{28}$ The findings of our study confirm these diverging perspectives and add to the present evidence by elaborating and clarifying how diversity in HCPs' clinical reasoning may lead to different decisionmaking in reablement.

In the first subtheme, a core concern was to improve physical capacity, which was considered essential for the participants' function in everyday living. Within this subtheme, research evidence about PA and its impact on physical capacity and function played an important role, which is consistent with other research evidence supporting this relationship. ${ }^{14-16}$ To overcome barriers to PA, HCPs not only found it valuable to use research-based exercise strategies but also to consider contextual premises and individual motivational factors that influence PA habits. ${ }^{22}$ These findings support the recently updated guidelines of PA presented by the $\mathrm{WHO}^{18}$ and exemplify how promoting PA can be integrated in a person-centered way through a publicly financed healthcare setting. However, the organizational circumstances of reablement were central in the HCPs' clinical reasoning, suggesting that different organizational premises influence their decision-making. Also, the HCPs suggested that establishing continued PA habits after reablement was a core challenge, particularly if the participants did not find PA and exercise activities sufficiently meaningful in their everyday living.

In the second subtheme, the participant's performance of the particular goal activities was a core focus of the HCPs' clinical reasoning. This subtheme shares similarities with the theory presented through the Canadian Model of Occupational Performance and Engagement (CMOPE) ${ }^{39}$ and its associated measurement tool, the Canadian Occupational Performance Measure (COPM), which has been included in previous reablement studies. ${ }^{4,5}$ In the CMOP-E, a person's occupational performance and engagement are central and understood as the dynamic interplay between the person, the activity, and the environment. ${ }^{39}$ Consistent with the CMOP-E, the HCPs emphasize the need to ensure that the reablement activities are meaningful, and they found it essential to analyze and practice the activity in its rightful environment as key to improving the participant's self-efficacy and motivation to engage in activities. Within this subtheme, the HCPs did not refer to research evidence in their clinical reasoning, but, rather, they emphasized the importance of gaining comprehensive knowledge about the individual participants and their daily life, challenges, and interests, using the COPM as a tool to facilitate this process. Concerning how to increase PA among older adults, as recommended by the $\mathrm{WHO},{ }^{18}$ this perspective does not focus on the promotion of PA but offers a valuable approach to ensure meaningfulness and self-efficacy in daily activities. This may improve participants' general activity levels and reduce sedentary behavior, which is a recently added component of the PA recommendations from the $\mathrm{WHO}^{18}$ and an emphasized field of further research. ${ }^{40}$ However, a sole emphasis on the perspectives in subtheme two may neglect considerations related to the participants' body functions and structures and overlook the benefits that can be reached by promoting PA or addressing the risks of not being sufficiently physically active in older age.

The overall clinical reasoning described in this study embraces all three levels of the ICF-model, involving considerations of body functions and structures, activity, and participation, and also considers interrelationships 
between health condition, environmental factors and personal factors, as outlined in the ICF-framework. ${ }^{13}$ The first subtheme identified in our study is particularly focused on body functions and structures, while the second subtheme is particularly targeted toward participation. However, both share a common focus in their emphasis on function in (everyday) activities as central in reablement. The findings of this study further suggest that to meet these different levels of functioning, HCPs have different priorities in their clinical reasoning, while building upon different types of knowledge.

Although it could be obvious to consider that the two subthemes may relate to the different disciplinary backgrounds of the HCPs, we did not find this association in our study. Rather, the HCPs seemed to have developed mutually agreed upon concepts within each reablement team, predominately emphasizing one of the two subthemes when deciding upon the primary content of reablement interventions. This joint agreement within the teams aligns with findings by Gabbay and LeMay, suggesting that HCPs, rather than working directly from theories or scientific evidence, develop "mindlines" of reasoning based on collectively reinforced, internalized, and tacit knowledge. ${ }^{41}$ However, if such mindlines and perspectives remain unspoken in daily practice, there is a risk of inadequate integration of different professional perspectives in the decision-making. In all of the municipalities included in this study, PTs and OTs were considered the "motors" of reablement and had the primary responsibility of developing the reablement plan. Although the competencies of healthcare assistants and RNs were considered highly valuable in reablement, this organization may have prevented the integration of theoretical perspectives from other disciplines in reablement delivery. Valuable perspectives and knowledge added by different disciplinary groups involved in reablement should therefore be further explored in future research.

This study was conducted in a Norwegian reablement setting, which likely has similarities as well as differences from other reablement settings around the world. Both PTs and OTs, home care staff, and, to some extent, RNs were included in the reablement settings included in our study, similar to reablement studies from other countries including Australia, ${ }^{42}$ Sweden, ${ }^{43}$ Denmark, ${ }^{44}$ and Japan. ${ }^{45}$ In the United Kingdom, however, reablement is provided through social services, and mainly social workers, and, to a lesser degree, OTs are reported to be involved in reablement. ${ }^{46-48}$ Contextual differences related to disciplinary groups involved, organization of reablement, and available resources may introduce different priorities and competencies in reablement practice and should be further explored.

\section{Strengths and Limitations}

A strength of this study was that we included a heterogenic sample of HCPs recruited from municipalities of different sizes and organization models. This allowed us to explore essential components of the HCPs' clinical reasoning despite some variations in reablement settings. However, as we found little evidence in the literature of which contextual factors influence reablement provision, there may be other influencing characteristics that we did not consider in our sampling strategy, which potentially could have introduced other perspectives.

The study was further strengthened by a thorough data analysis, which was critically discussed by a team of researchers with different educational backgrounds and expertise. Also, we believe the use of the COREQ checklist $^{33}$ and Elo et al's reflection questions for increasing trustworthiness ${ }^{38}$ improved the methodological stringency and trustworthiness of this study. Study limitations were that we did not gain feedback on the findings from the HCPs we interviewed, which could have further strengthened the trustworthiness of the findings. Although we encouraged the HCPs to share and elaborate on specific examples from their practice, we are aware that the core clinical reasoning in their everyday practice is contextually dependent and may withhold nuances and dynamics that are not captured in this study.

\section{Implications for Practice}

By exploring HCPs' clinical reasoning and perspectives regarding PA, this study may explain some of the underlying and unspoken assumptions that may remain in interdisciplinary reablement collaborations. The findings can be used to facilitate critical discussions and assist HCPs in expressing and critically reviewing underlying perspectives regarding PA in their practice. They also point out the need for close interdisciplinary collaborations that enable an appropriate integration of different knowledge to ensure a broad foundation for evidence-based practice and the ability to meet different needs of the participants.

The findings contribute to an improved understanding of the knowledge used to promote PA in a person-centered way in a community healthcare setting, thus informing policymakers, leaders, and clinicians. By making the HCPs clinical reasoning more explicit, the findings may 
also elucidate types of knowledge that should be included in training of HCPs in reablement.

\section{Implications for Research}

The findings of this study demonstrate that PA can be a part of reablement delivery. This may add to the previous understanding and perspectives on the concept of reablement, proposing strategies for how reablement can target body functions and structures, in addition to activity and participation, in order to improve older adults' function. Future studies should further explore how PA is integrated in an appropriate, feasible, effective and meaningful way to improve and maintain participants functional ability. We suggest that future research also explore how the context and organizational setting enables or hinders the facilitation of PA in reablement.

Our findings suggest that there are different theoretical perspectives influencing reablement strategies. We recommend more focus be placed on elaborating and clarifying such perspectives, in order to make a stronger theoretical foundation of reablement, and also informing how these perspectives and components can complement each other and contribute to reaching the goals of reablement. This may also inform which professions are beneficial to include, and what knowledge is required for home care assistants to deliver reablement at a high level. By gaining better insight into HCPs clinical reasoning processes, researchers have an improved understanding of how PA is being used in practice, which may better inform about the type of future research needed to further develop reablement.

\section{Conclusion}

The findings of this study suggest that HCPs build their clinical reasoning from different knowledge and perspectives, leading to diverse priorities on how to integrate PA in reablement. Such underlying theoretical perspectives may lead to conflicting decision-making in reablement, but they also may integrate different perspectives in a complementary and dynamic way that adequately meets the individual preferences and needs of participants. The potential of promoting PA through reablement, based on health policy recommendations, seems to be influenced by theoretical perspectives and interdisciplinary collaboration and negotiation between HCPs. There is a need to further investigate how the promotion of PA can be efficiently integrated into the context of reablement, how it meets the needs and preferences of participants, and which contextual factors influence PA promotion in reablement.

\section{Abbreviations}

PA, physical activity; HCP, health care professional; PT, physiotherapist; OT, occupational therapist; RN, registered nurse; COPM, Canadian Occupational Performance Measure.

\section{Data Sharing Statement}

The data of this study will not be shared due to the risk of reducing the confidentiality of the participants of the study.

\section{Ethics Approval and Informed Consent}

The study was not considered by the Regional Ethics Committee to be within their field of medical and health research ethics approval, since no health data was collected. The study received approval from the Norwegian Centre for Research Data (Ref. nr. 405436). All participants signed an informed consent before participating in the study, in which they accepted that their information would be used in scientific publications, but would remain anonymized.

\section{Acknowledgments}

We thank all of the HCPs who participated in the study for their willingness to participate and share their experiences. We also thank all of the leaders that assisted in recruiting HCPs for the study.

\section{Author Contributions}

All authors contributed to the development and planning of the study and made a significant contribution to the work reported. HLM carried out interviews, transcribed interview data, and was the main contributor in the analysis of data. All authors contributed to data analysis, drafted, substantially revised and critically reviewed the article. All authors agreed on the journal to which the article was submitted; gave final approval on all versions of the article prior to publication; and agree to take responsibility and be accountable for the contents of the article.

\section{Funding}

This study is part of a Ph.D. project undertaken by the first author, HLM, and funded by Nord University, Norway. The funders had no role in the study design, data 
collection and analysis, decision to publish, or preparation of the manuscript.

\section{Disclosure}

None of the authors participating in the study (HLM, CFM, EB, and LU) have any competing interests.

\section{References}

1. Metzelthin SF, Rostgaard T, Parsons M, Burton E. Development of an internationally accepted definition of reablement: a Delphi study. Ageing Soc. 2020;1-16. doi:10.1017/S0144686X20000999

2. Aspinal F, Glasby J, Rostgaard T, Tuntland H, Westendorp RG. New horizons: reablement - supporting older people towards independence. Age Ageing. 2016;45(5):572-576. doi:10.1093/ageing/afw094

3. Mjøsund HL, Moe CF, Burton E, Uhrenfeldt L. Integration of Physical Activity in reablement for community dwelling older adults: a systematic scoping Review. $J$ Multidiscip Healthc. 2020;13:1291-1315. doi:10.2147/JMDH.S270247

4. Tuntland H, Aaslund MK, Espehaug B, Forland O, Kjeken I. Reablement in community-dwelling older adults: a randomised controlled trial. BMC Geriatr. 2015;15(1):145. doi:10.1186/s12877-015$0142-9$

5. Langeland E, Tuntland H, Folkestad B, Førland O, Jacobsen FF, Kjeken I. A multicenter investigation of reablement in Norway: a clinical controlled trial. BMC Geriatr. 2019;19(1):29. doi:10.1186/ s12877-019-1038-x

6. Lewin G, De San Miguel K, Knuiman M, et al. A randomised controlled trial of the home independence program, an Australian restorative home-care programme for older adults. Health Soc Care Community. 2013;21(1):69-78. doi:10.1111/j.1365-2524.2012.01088.x

7. Langeland E, Førland O, Aas E, et al. 2016. Modeller for hverdagsrehabilitering - en følgeevaluering i norske kommuner [Models of reablement - an evaluation in Norwegian municipalities]. In: Senter for Omsorgsforskning, Rapportserie Nr 6/2016; Available from: http://hdl.handle.net/11250/2389813.

8. Eliassen M, Henriksen NO, Moe S. Physiotherapy supervision of home trainers in interprofessional reablement teams. $J$ Interprof Care. 2018;1-7.

9. Hjelle KM, Skutle O, Alvsvåg H, Førland O. Reablement teams' roles: a qualitative study of interdisciplinary teams' experiences. $J$ Multidiscip Healthc. 2018;11:305-316. doi:10.2147/JMDH. S160480

10. Cochrane A, Furlong M, McGilloway S, Molloy DW, Stevenson M, Donnelly M. Time-limited home-care reablement services for maintaining and improving the functional independence of older adults. Cochrane Database Syst Rev. 2016;10:Cd010825. doi:10.1002/ 14651858.CD010825.pub2

11. Legg L, Gladman J, Drummond A, Davidson A. A systematic review of the evidence on home care reablement services. Clin Rehabil. 2016;30(8):741-749. doi:10.1177/0269215515603220

12. Pettersson C, Iwarsson S. Evidence-based interventions involving occupational therapists are needed in reablement for older community-living people: a systematic review. Br J Occup Ther. 2017;80(5):273-285. doi:10.1177/0308022617691537

13. World Health Organization. ICF - Towards a common language for functioning, disability and health. Geneva; 2002. Available from: http://www.who.int/classifications/icf/icfbeginnersguide.pdf?ua=1. Accessed April 11, 2021.

14. Chou CH, Hwang CL, Wu YT. Effect of exercise on physical function, daily living activities, and quality of life in the frail older adults: a meta-analysis. Arch Phys Med Rehabil. 2012;93(2):237-244. doi:10.1016/j.apmr.2011.08.042
15. El-Khoury F, Cassou B, Charles MA, Dargent-Molina P. The effect of fall prevention exercise programmes on fall induced injuries in community dwelling older adults: systematic review and meta-analysis of randomised controlled trials. BMJ. 2013;347: f6234. doi:10.1136/bmj.f6234

16. Lozano-Montoya I, Correa-Perez A, Abraha I, et al. Nonpharmacological interventions to treat physical frailty and sarcopenia in older patients: a systematic overview - the SENATOR project ONTOP series. Clin Interv Aging. 2017;12:721-740. doi:10.2147/CIA.S132496

17. The Norwegian Directorate of Health. Nasjonale faglige råd: fysisk aktivitet for voksne og eldre. [National recommendations: physical activity for adults and older adults] [internet]. Available from: https:// www.helsedirektoratet.no/faglige-rad/fysisk-aktivitet-for-barn-ungevoksne-eldre-og-gravide/fysisk-aktivitet-for-voksne-og-eldre. Accessed April 11, 2021.

18. World Health Organization. WHO guidelines on physical activity and sedentary behaviour. Geneva; 2020. Available from: https://www. who.int/publications/i/item/9789240015128. Accessed April 11, 2021.

19. World Health Organization. Physical activity strategy for the WHO European Region 2016-2025. Regional office for Europe 2016. Available from: http://www.euro.who.int/_data/assets/pdf_file/0014/ 311360/Physical-activity-strategy-2016-2025.pdf?ua=1. Accessed April 11, 2021.

20. Gomes M, Figueiredo D, Teixeira L, et al. Physical inactivity among older adults across Europe based on the SHARE database. Age Ageing. 2017;46(1):71-77. doi:10.1093/ageing/afw165

21. Burton E, Lewin G, Boldy D. Barriers and motivators to being physically active for older home care clients. Phys Occup Ther Geriatr. 2013;31(1):21-36. doi:10.3109/02703181.2012.751474

22. Devereux-Fitzgerald A, Powell R, Dewhurst A, French DP. The acceptability of physical activity interventions to older adults: a systematic review and meta-synthesis. Soc Sci Med. 2016;158:14-23. doi:10.1016/j.socscimed.2016.04.006

23. Birkeland A, Tuntland H, Forland O, Jakobsen FF, Langeland E. Interdisciplinary collaboration in reablement - a qualitative study. J Multidiscip Healthc. 2017;10:195-203. doi:10.2147/JMDH.S133417

24. Gustafsson L, Anbäcken E, Elfström M, Zander V, Östlund G. Working with short-term goal-directed reablement with older adults: strengthened by a collaborative approach. Nord J Nurs Res. 2019;2057158519850974.

25. Jokstad K, Skovdahl K, Landmark BT, Haukelien H. Ideal and reality; Community healthcare professionals' experiences of userinvolvement in reablement. Health Soc Care Community. 2019;27 (4):907-916. doi:10.1111/hsc.12708

26. Jordan Z, Lockwood C, Munn Z, Aromataris E. Redeveloping the JBI model of evidence based healthcare. JBI Evidence Implement. 2018;16(4):227-241.

27. Higgs J, Jensen GM, Loftus S, Christensen N. Clinical Reasoning in the Health Professions. 4th ed. Edinburgh: ElsevierHealth Sciences; 2018.

28. Eliassen M, Lahelle A. Enhancing functional improvement in reablement - a qualitative study. Eur J Physiother. 2020;1-7. doi:10.1080/ 21679169.2020.1761449

29. Gustafsson LK, Ostlund G, Zander V, Elfstrom ML, Anbacken EM. 'Best fit' caring skills of an interprofessional team in short-term goaldirected reablement: older adults' perceptions. Scand J Caring Sci. 2019;33(2):498-506. doi:10.1111/scs.12650

30. Moe A, Brataas HV. Interdisciplinary collaboration experiences in creating an everyday rehabilitation model: a pilot study. $J$ Multidiscip Healthc. 2016;9:173-182. doi:10.2147/JMDH.S103696

31. Hjelle KM, Skutle O, Førland O, Alvsvåg H. The reablement team's voice: a qualitative study of how an integrated multidisciplinary team experiences participation in reablement. $J$ Multidiscip Healthc. 2016;9:575-585. doi:10.2147/JMDH.S115588 
32. Maxwell J. A Realist Approach to Qualitative Research. Sage; 2012.

33. Tong A, Sainsbury P, Craig J. Consolidated criteria for reporting qualitative research (COREQ): a 32-item checklist for interviews and focus groups. Int J Qual Health Care. 2007;19(6):349-357. doi:10.1093/intqhe/mzm042

34. Patton MQ. Qualitative Research \& Evaluation Methods: Integrating Theory and Practice. 4th ed. Los Angeles: Sage; 2015.

35. Eliassen M, Henriksen No M, Moe S. Variations in physiotherapy practices across reablement settings. Physiother Theory Pract. 2018;36(1):108-121. doi:10.1080/09593985.2018.1481162

36. Braun V, Clarke V. To saturate or not to saturate? Questioning data saturation as a useful concept for thematic analysis and sample-size rationales. Qual Res Sport Exerc Health. 2021;13(2):201-216. doi:10.1080/2159676X.2019.1704846

37. Erlingsson C, Brysiewicz P. A hands-on guide to doing content analysis. Afr J Emerg Med. 2017;7(3):93-99. doi:10.1016/j. afjem.2017.08.001

38. Elo S, Kääriäinen M, Kanste O, Pölkki T, Utriainen K, Kyngäs H. Qualitative content analysis: a focus on trustworthiness. SAGE Open. 2014;4(1):1. doi:10.1177/2158244014522633

39. Townsend EA, Polatajko HJ. Enabling Occupation II: Advancing an Occupational Therapy Vision for Health, Well-Being, \& Justice Through Occupation. Canadian Association of Occupational Therapists; 2007.

40. Dogra S, Ashe MC, Biddle SJH, et al. Sedentary time in older men and women: an international consensus statement and research priorities. Br J Sports Med. 2017;51(21):1526-1532. doi:10.1136/ bjsports-2016-097209

41. Gabbay J, le May A. Evidence based guidelines or collectively constructed "mindlines?" Ethnographic study of knowledge management in primary care. BMJ. 2004;329(7473):1013. doi:10.1136/ bmj.329.7473.1013
42. Burton E, Lewin G, Clemson L, Boldy D. Effectiveness of a lifestyle exercise program for older people receiving a restorative home care service: a pragmatic randomized controlled trial. Clin Interv Aging. 1591-1601;2013(8).

43. Zingmark M, Evertsson B, Haak M. Characteristics of occupational therapy and physiotherapy within the context of reablement in Swedish municipalities: a national survey. Health Soc Care Community. 2020;28(3):1010-1019. doi:10.1111/hsc.12934

44. Bodker MN, Langstrup H, Christensen U. What constitutes 'good care' and 'good carers'? The normative implications of introducing reablement in Danish home care. Health Soc Care Community. 2019;27(5):e871-e878. doi:10.1111/hsc.12815

45. Hattori S, Yoshida T, Okumura Y, Kondo K. Effects of reablement on the independence of community-dwelling older adults with mild disability: a randomized controlled trial. Int J Environ Res Public Health. 2019;16(20):3954. doi:10.3390/ijerph16203954

46. Whitehead PJ, Walker MF, Parry RH, Latif Z, McGeorge ID, Drummond AER. Occupational Therapy in HomEcare Re-ablement Services (OTHERS): results of a feasibility randomised controlled trial. BMJ Open. 2016;6(8):e011868. doi:10.1136/bmjopen-2016011868

47. Slater P, Hasson F. An evaluation of the reablement service programme on physical ability, care needs and care plan packages. J Integr Care. 2018;26(2):140-149. doi:10.1108/JICA-11-2017-0042

48. Beresford B, Mayhew E, Duarte A, et al. Outcomes of reablement and their measurement: findings from an evaluation of English reablement services. Health Soc Care Community. 2019;27 (6):1438-1450. doi:10.1111/hsc. 12814
Journal of Multidisciplinary Healthcare

\section{Publish your work in this journal}

The Journal of Multidisciplinary Healthcare is an international, peerreviewed open-access journal that aims to represent and publish research in healthcare areas delivered by practitioners of different disciplines. This includes studies and reviews conducted by multidisciplinary teams as well as research which evaluates the results or conduct of such teams or healthcare processes in general. The journal covers a very wide range of areas and welcomes submissions from practitioners at all levels, from all over the world. The manuscript management system is completely online and includes a very quick and fair peer-review system. Visit http://www.dovepress.com/testimonials. php to read real quotes from published authors. 